\title{
Eventos adversos na terapia farmacológica pediátrica: revisão integrativa da literatura
}

\author{
Adverse events in pediatric pharmacotherapy: integrative literature review
}

Acontecimientos adversos en terapia farmacológica pediátrica: revisión integrativa de la literatura

\section{Tathiana Silva de Souza Martins', Zenith Rosa Silvino"', Luciana Rodrigues da Silva"I' \\ 'Universidade do Estado do Rio de Janeiro, Hospital Universitário Pedro Ernesto. Niterói-RJ, Brasil. Universidade Estácio de Sá, Curso de Graduação em Enfermagem. Rio de Janeiro-RJ, Brasil. \\ " Universidade Federal Fluminense, Escola de Enfermagem Aurora de Afonso Costa, \\ Departamento de Fundamentos de Enfermagem e Administração. Niterói-RJ, Brasil. \\ III Universidade Federal Fluminense, Escola de Enfermagem Aurora de Afonso Costa, Departamento de Enfermagem Materno Infantil e Psiquiatria. Niterói-RJ, Brasil.}

Submissão: 06/01/2010 Revisão: 06/09/2010 Aprovação: 07/11/2010

\section{RESUMO}

Trata-se de uma revisão integrativa da literatura realizada em bases de dados online, no período entre 2003-2009, com o objetivo de identificar as evidências disponíveis sobre os principais eventos adversos relacionados a medicamentos na clientela pediátrica e propor estratégias para a redução dos mesmos. Evidenciou-se a inadequação da apresentação dos fármacos direcionada ao atendimento das necessidades terapêuticas específicas das crianças. Entende-se que a tomada de decisão do enfermeiro sobre o aprazamento seguro, o preparo e a administração de fármacos, deve estar baseada em avaliação criteriosa e individualiza de cada cliente. Assim, é necessário que o profissional esteja embasado cientificamente, para implementar intervenções eficazes que sejam capazes de reduzir e, até mesmo, evitar os eventos adversos oriundos da uni ou polifarmácia. Descritores: Enfermagem; Enfermagem Pediátrica; Erros de medicação; Interações de medicamentos.

\section{ABSTRACT}

This paper is about an integrative review from literature realized in databases online, into the period among 2003-2009, with the objective of identify the available evidence on the main drug-related adverse events in pediatric clientele and propose strategies for reducing them. The study revealed the inadequacy of the drugs presentation to meet the specific therapeutic needs of children. It is understood that the nurse's decision making about safe preparation and administration of drugs should be based on careful and individualized assessment of each client. It is therefore necessary that the professional be scientifically supported, to implement effective interventions that are able to reduce and even prevent adverse events from the uni or polypharmacy.

Key words: Nursing; Pediatric Nursing; Medication errors; Drug interactions.

\section{RESUMEN}

Se trata de una revisión integrativa de la literatura, Ilevada a cabo en bases de datos en línea, en el período de 2003 a 2009 , con el objetivo de identificar la evidencia disponible sobre los principales eventos adversos relacionados a los medicamentos en la clientela de pediatría, y proponer estrategias para su reducción. Identificó-se la presentación inadecuada de los fármacos para satisfacer las necesidades terapéuticas específicas de los niños. Se entiende que la toma de decisiones de la enfermera sobre la preparación y la administración de fármacos debe basarse en una evaluación cuidadosa e individualizada de cada cliente. Por tanto, es necesario que el profesional se apoye científicamente, para ejecutar intervenciones eficaces que sean capaces de reducir e incluso prevenir los efectos adversos de la uni o de la polifarmacia.

Palabras clave: Enfermería; Enfermería Pediátrica; Errores de medicación; Interacciones medicamentosas. 


\section{INTRODUÇÃO}

Se por um lado os medicamentos têm a possibilidade de solucionar vários problemas de saúde, aumentando a expectativa de vida, erradicando certas doenças e minimizando o sofrimento dos indivíduos, por outro, podem contribuir para o aumento dos custos em saúde se utilizados inadequadamente e/ou levar à ocorrência de eventos adversos ${ }^{(1)}$.

O termo evento adverso (EA) refere-se ao aparecimento de um problema de saúde causado pelo cuidado e não pela doença de base, ou seja, uma lesão não intencional que resultou em incapacidade temporária ou permanente e/ou prolongamento do tempo de permanência ou morte como conseqüência do cuidado prestado. São observados eventos adversos oriundos de procedimentos cirúrgicos, utilização de medicamentos, procedimentos médicos, tratamento não-medicamentoso, demora ou incorreção no diagnóstico. Os eventos adversos relacionados a medicamentos são responsáveis por cerca de $20 \%$ do total de casos observados, atrás apenas daqueles associados a procedimentos cirúrgicos ${ }^{(2)}$.

Desta forma, fica claro que em um EA relacionado a Medicamentos (EAM) deve-se considerar tanto a eficácia e a segurança intrínseca do medicamento, como todos os procedimentos envolvidos no processo de utilização. Surge, então, a necessidade de diferenciar o componente responsável pelo EAM, que são subdivididos em dois grupos: reações adversas a medicamentos (RAM) e erros de medicação. O primeiro diz respeito ao risco inerente frente à utilização adequada de medicamentos, portanto, inevitáveis. Os erros de medicação são entendidos como qualquer evento passível de prevenção, decorrentes do uso inadequado, ou não; portanto, possivelmente relacionado com falhas nos procedimentos ${ }^{(3)}$.

No tratamento de crianças com doenças agudas e crônicas, a administração de medicamentos demonstra, cada vez mais, destacada relevância clínica. No entanto, para a implementação segura da terapia medicamentosa, enfermeiros devem ter conhecimentos sobre o correto preparo e administração de fármacos e soluções, pois a partir da prescrição médica, executam o aprazamento da administração, prescrevem cuidados concernentes à administração e monitorização, conforme o tipo de terapêutica, e registram a resposta do paciente ao tratamento, incluindo possíveis eventos adversos relacionados ao uso de fármacos e soluções ${ }^{(4)}$.

Alguns fármacos são liberados no mercado sem benefício definido, ou com limitada experiência de utilização na clientela pediátrica, o que torna a questão relacionada à ocorrência de EAM ainda mais delicada ${ }^{(5)}$. Essa deficiência causa, com freqüência, a prescrição de fármacos fora dos termos de licença do produto (off-label), aumentando os riscos de intoxicação dos pacientes pediátricos. Medicamentos off-label são aqueles cuja utilização não está coberta pela licença do produto, seja: administração de doses maiores ou em maior frequência, administração de medicamento para indicação não descrita na licença, administração de medicamento em crianças cuja faixa etária não é coberta pela licença do fármaco, utilização de vias alternativas de administração ou utilização do medicamento em situações na qual ele é contra indicado(6).
Procurando contribuir e somar esforços para a melhoria da assistência de enfermagem prestada à criança em uso de terapia medicamentosa, a presente investigação teve como objetivo identificar as evidências disponíveis na literatura sobre os principais EAM na clientela pediátrica e propor estratégias para a redução dos mesmos.

Na presente pesquisa, selecionou-se como método um dos recursos da prática baseada em evidencias, ou seja, a revisão integrativa da literatura, a qual possibilita a síntese e a analise do conhecimento científico já produzido sobre o tema investigado.

A prática, baseada em evidências, movimento estruturado como facilitador da tomada de decisão, pode ser definida como um processo de busca, avaliação e aplicação de evidências científicas para o tratamento e gerenciamento da saúde ${ }^{(7)}$. É uma abordagem que possibilita a melhoria da qualidade da assistência prestada ao paciente e incentiva o profissional de saúde a buscar conhecimento cientifico por meio do desenvolvimento de pesquisas ou aplicação na sua prática dos resultados encontrados na literatura ${ }^{(8)}$.

Esse movimento é um dos precursores da enfermagem baseada em evidencias, a qual pode ser definida como uso explicito e judicioso das informações obtidas em pesquisa, derivada de teoria para a tomada de decisões sobre o cuidado dispensado a indivíduos ou grupos de pacientes, considerando as necessidades e preferências individuais ${ }^{(7)}$ e tem sido intensamente discutida nas ultimas décadas, na busca da delimitação de suas potencialidades e limitações.

\section{METODOLOGIA}

Para a elaboração da presente revisão integrativa as seguintes etapas foram percorridas: determinação dos critérios de inclusão e exclusão de artigos (seleção da amostra); definição das informações a serem extraídas dos artigos e teses do banco da Universidade de São Paulo (USP) selecionados; análise dos resultados; discussão e apresentação dos resultados. Para guiar a revisão integrativa, formulou-se a seguinte questão: quais são os principais eventos adversos relacionados à terapia farmacológica em pediatria?

Para a seleção dos artigos foram utilizadas as seguintes bases de dados: LILACS, MEDLINE, ADOLEC, BBO, BDENF, DESASTRES, HOMEOINDEX, MEDCARIBE, REPIDISCA, PAHO e WHOLIS. Para a seleção de teses e dissertações foi utilizado o banco de dados da USP. Dessa forma, procurou-se ampliar o âmbito da pesquisa, minimizando possíveis vieses nessa etapa do processo de elaboração da revisão integrativa.

Nessas bases de dados, foram encontrados artigos segundo os critérios de inclusão definidos para a presente revisão integrativa, que foram: artigos publicados na íntegra online em português e espanhol, no período compreendido entre 2003-2009; artigos publicados cuja metodologia adotada permitisse obter os principais eventos adversos associados à terapia farmacológica em pediatria. Os descritores utilizados foram: "erros de medicação", "interações de medicamentos" e "enfermagem pediátrica". Vale mencionar que esses foram os descritores selecionados, pelo fato das palavras "evento adverso" e "reações adversas a medicamentos" 
não constarem como descritores nas bases de dados. A busca foi realizada pelo acesso online e, utilizando os critérios de inclusão, a amostra final da pesquisa foi constituída três (3) artigos e três (3) teses da USP.

Ressalta-se que as três (3) teses da USP não contemplavam a faixa etária da amostra estudada. Contudo, as mesmas foram inseridas na presente revisão por apresentarem questões importantes e intrínsecas aos eventos adversos relacionados a medicamentos, independentemente da idade.

Para a coleta de dados do material que foi incluído na revisão integrativa, foi elaborado um instrumento, que contemplou os seguintes itens: Identificação do artigo/resumo original; propósito do estudo; tamanho da amostra, características e cenário; desenho da pesquisa e instrumento; principais achados e conclusões/recomendações.

A apresentação dos resultados e discussão dos dados obtidos foi feita de forma descritiva, possibilitando ao leitor a avaliação da aplicabilidade da revisão integrativa elaborada, de forma a atingir o objetivo desse método, ou seja, impactar positivamente na qualidade da prática de enfermagem, fornecendo subsídios ao enfermeiro na sua tomada de decisão.

\section{RESULTADOS E DISCUSSÕES}

Todos os artigos e teses incluídos na revisão integrativa são de autoria de Enfermeiros. Os três (3) artigos analisados foram escritos por Enfermeiros docentes da Universidade Federal de São Paulo (UNIFESP); e as três (3) teses, são de autoria de Enfermeiros assistenciais. Em relação ao tipo de revista nas quais foram publicados os artigos incluídos na revisão, todos foram publicados em revistas de Enfermagem geral. Quanto ao tipo de delineamento de pesquisa nos artigos e teses avaliados, encontrou-se, na amostra: dois estudos descritivo-exploratórios, um estudo quase-experimental, dois estudos transversais e um estudo observacional e transversal. A seguir, apresenta-se a síntese dos três artigos e das três teses, respectivamente:

1. Yamanaka TI, Pereira DG, Pedreira MLG, Peterlini MAS ${ }^{(4)}$. Redesenho das atividades de enfermagem para redução de erros de medicação em pediatria. Objetivo: verificar a influência de uma intervenção de reestruturação de atividades, composta pela elaboração de um algoritmo e desenvolvimento de um programa de educação, com vistas à redução de erros de medicação em três unidades de pediatria de um hospital universitário, a partir de pesquisa anteriormente realizada nas mesmas unidades. Principais resultados: Foi encontrada diferença estatisticamente significante entre as avaliações antes e após a intervenção, quanto à distribuição dos tipos de erros de medicação ( $p$ < 0,0001). Em parte dos erros de medicação, a proporção encontrada na avaliação anterior foi significantemente maior do que na avaliação posterior, a saber: omissão da dose $(p<0,0001)$, medicação suspensa pelo médico e não registrada como suspensa pela enfermagem $(p<0,0001)$ e hora errada ( $p=0,0002)$. Recomendações/ Conclusões: $A$ mudança de comportamento é um processo lento e que exige constante investimento para sua obtenção, sendo necessário promover continuidade no processo de mudança. A conscientização e o aprendizado por parte da equipe de enfermagem envolvida na execução da terapia medicamentosa devem ser conquistados de forma gradativa e, para que isso aconteça, é importante realizar atividades que analisem e promovam melhorias no sistema de medicação, sendo que as estratégias de educação sobre a importância do tema devem preceder, em determinadas situações, as intervenções de melhoria a fim de motivar a equipe para o alcance de melhores resultados.

2. Peterlini MAS, Chaud MN, Pedreira MLG ${ }^{(9)}$. Órfãos de terapia medicamentosa: a administração de medicamentos por via intravenosa em crianças hospitalizadas. Objetivos: Verificar o número de medicamentos administrados; Identificar e classificar farmacologicamente os medicamentos administrados por via intravenosa mais utilizados; Verificar a adequação da apresentação farmacológica para o uso em pediatria e Estimar o custo da administração de algumas drogas utilizadas para terapia intravenosa em crianças. Principais resultados: Obteve-se uma população de 1212 prescrições e média diária, nas quatro unidades investigadas, de 40,4 crianças submetidas à terapia intravenosa. Dentre os 41 tipos de medicamentos administrados por via intravenosa identificados, nenhum possuía apresentação direcionada ao atendimento de necessidades terapêuticas específicas de crianças. Os medicamentos comercializados em ampolas, como amicacina, furosemida, midazolan e ranitidina, apresentam concentração e dosagem superior à utilizada em cada dose pediátrica, principalmente em crianças até três anos de idade, faixa etária que se caracteriza como a que mais tem tido necessidade de assistência hospitalar, e assim, como todo o excedente da droga deve ser desprezado após a administração, ocorre um considerável aumento no custo do tratamento proposto. Outro aspecto a considerar, refere-se a drogas como metilpredinosolona e fentanil, cuja concentração por mililitro de solução é muito superior à dose máxima recomendada por dia. Recomendações/ Conclusões: A totalidade, 41, dos medicamentos identificados não tinha apresentação farmacológica pediátrica. O custo estimado da terapia medicamentosa intravenosa em uma criança, em 24 horas, foi de U\$46.23, sendo que U\$ 6.71 de medicamentos foram administrados e \$ 39.52 desprezados, por excederem as necessidades terapêuticas diárias da criança. Observou-se que a comercialização em ampolas também é fator que contribui para o aumento indireto dos custos.

3. Peterlini MAS, Chaud MN, Pedreira MLG ${ }^{(10)}$. Incompatibilidade entre fármacos e materiais de cateteres e acessórios da terapia intravenosa. Objetivo: identificar a ocorrência de incompatibilidade entre fármacos e materiais de confecção de cateteres e acessórios da terapia intravenosa administrada em crianças hospitalizadas, em quatro unidades pediátricas de um hospital universitário. Principais resultados: Foram identificadas 519 execuções de terapia 
intravenosa em 178 crianças. Os fármacos foram administrados por 209 cateteres, confeccionados em vialon, teflon, aço inoxidável e poliuretano. O polivinil cloreto foi material de confecção de todos (601) os acessórios utilizados para infusão de fármacos, sendo 60,7\% associações compatíveis, 19,8\% incompatíveis e para 19,5\% observou-se a falta de evidências científicas que respaldem esta prática da enfermagem. Recomendações/ Conclusões: Os autores concluíram que das 519 execuções de fármacos por meio de cateteres intravenosos, 3,1\% foram incompatíveis com o material dos dispositivos e em $96,9 \%$ das administrações observadas não foi encontrada referência na literatura consultada sobre a incompatibilidade. Desta forma, os resultados obtidos permitem deduzir que a ocorrência de incompatibilidades, como também a falta de evidências científicas, são dificuldades cotidianas presentes na prática da enfermeira pediatra durante a administração de medicamentos.

4. Lima REF, Cassiani $\mathrm{SHB}^{(11)}$. Interações medicamentosas potenciais em pacientes de Unidade de Terapia Intensiva de um Hospital Universitário do Ceará. Objetivo: Analisar as potenciais interações medicamentosas em pacientes de uma Unidade de Terapia Intensiva de um Hospital Universitário do Ceará. Principais resultados: Foram selecionados 102 pacientes, destes 74, apresentaram 311 potenciais interações medicamentosas. Quanto ao número de medicamentos, os pacientes que apresentaram interações medicamentosas receberam mais do que o dobro de medicamentos quando comparados aos pacientes sem interação medicamentosa. As interações medicamentosas foram mais freqüentes entre os medicamentos do sistema nervoso. Verificou-se que dos 1845 medicamentos analisados, 1140 foram aprazados para o mesmo horário, sendo o horário das 06 horas o que concentrou o maior numero de medicamentos administrados. Recomendações/ Conclusões: Os autores salientam que $80 \%$ das ações para minimizar ou até evitar os efeitos indesejáveis das interações medicamentosas podem ser realizados pelo enfermeiro. No entanto, para que as intervenções ocorram de fato é importante que o enfermeiro tenha conhecimento quanto aos mecanismos farmacológicos das interações medicamentosas, bem como seus fatores precipitantes.

5. Pelliciotti JSS, Kimura $\mathbf{M}^{(12)}$. Qualidade de vida relacionada à saúde de profissionais de enfermagem e erros de medicação em Unidades de Terapia Intensiva. Objetivos: Caracterizar os erros de medicação relatados por profissionais de enfermagem que atuam em UTI; Comparar os dados sócio-demográficos, do trabalho e da qualidade de vida relacionada à saúde (QVRS) dos profissionais envolvidos e não envolvidos com erros de medicação em UTI; Verificar se os domínios da QVRS são fatores independentes associados aos erros de medicação em UTI. Principais resultados: Entre os 18 profissionais que mencionaram ter cometido o erro, seis eram enfermeiros e doze técnicos de enfermagem. Houve notificação do erro em $61 \%$ dos casos. Os erros mais freqüentes foram os da fase de administração. Aspectos emocionais e os turnos de trabalho da tarde e da noite mostraram associação significativa com o erro de medicação. Recomendações/ Conclusões: Os resultados desse estudo trazem subsídios para a adoção de medidas institucionais especificamente direcionadas às necessidades dos profissionais de enfermagem, contribuindo, desta forma, para uma assistência mais segura aos pacientes.

6. Optiz SP, Cassiani SHB ${ }^{(13)}$. Sistema de medicação: análise dos erros nos processos de preparo e administração de medicamentos em um hospital de ensino. Objetivos: Identificar, analisar e comparar os erros de medicação ocorridos nos processos de preparo e administração em uma unidade de internação clínica de um hospital público de ensino. Principais resultados: A amostra foi constituída de 1.129 doses de medicamentos. Verificou-se que 866 prescrições estavam manuscritas, 126 não continham o nome legível do medicamento, em 267 faltavam as doses, em 107 não constava a via, em 112 não havia a forma de apresentação, em 20 faltava a frequência e em 338 não constavam o tipo e volume do diluente para o preparo. No preparo de medicamentos, foi identificado que 976 doses estavam rotuladas incorretamente e 49 doses não possuíam rotulo. Nas observações em que se desconhecia previamente a prescrição os erros mais ocorrentes foram: 130 erros de horário, 71 erros de omissão e 41 erros de dose. Nas observações em que se conhecia previamente a prescrição os erros mais ocorrentes foram: 85 erros de horário e 35 erros de omissão. Recomendações/ Conclusões: Os autores propõem como medidas para a redução dos erros nesta instituição a formação de um grupo multiprofissional com a finalidade de discutir e estabelecer estratégias que possam promover a segurança do paciente.

Apontou-se nos trabalhos encontrados na literatura que os EAM são do tipo: erro de medicação e reação adversa medicamentosa, esta relacionada especificamente as interações medicamentosas oriundas do aprazamento não seguro dos fármacos e da inexistência de drogas que possuam apresentação direcionada ao atendimento das necessidades terapêuticas das crianças.

Verificou-se que um artigo e duas teses abordavam os erros de medicação como uma questão inerente à equipe de enfermagem, atrelando a terapia medicamentosa à segurança do paciente ${ }^{(4,12,13)}$. Desta forma, é importante que o enfermeiro conheça as responsabilidades legais que envolvem a execução da terapia medicamentosa para promover a segurança do paciente e realizar cuidados competentes e adequados às necessidades do paciente e as características da terapia prescrita $^{(14)}$. E, neste sentido, a segurança do paciente deve ser uma prioridade a ser cumprida nas instituições hospitalares, a fim de minimizar riscos e falhas, e maximizar a satisfação da clientela.

Quanto ao EAM relacionado à incompatibilidade percebeu-se que um artigo relacionava tal ocorrência aos fármacos 
e materiais de confecção de cateteres e acessórios da terapia intravenosa ${ }^{(10)}$, enquanto uma tese tratava o tema relacionando-o às interações medicamentosas ${ }^{(11)}$. Sabe-se que a interação medicamentosa (IM) é considerada fenômeno que ocorre quando os efeitos de um fármaco são modificados devido à administração simultânea de outro fármaco ou alimento. Essa interação pode resultar na diminuição, anulação ou aumento do efeito de um dos fármacos ${ }^{(15)}$.

Embora nem todas as interações medicamentosas possam ser prevenidas, a difusão do conhecimento para os profissionais de saúde, quanto aos principais fatores de risco de interações medicamentosas, assim como o mecanismo de ação das interações, associados à divulgação das interações medicamentosas mais freqüentes e relevantes na prática clínica, constitui um dos principais instrumentos de prevenção das interações medicamentosas. Esse conhecimento permitirá aos profissionais de saúde optarem por regimes terapêuticos e horários de administração de medicamentos mais seguros conduzindo assim à assistência de qualidade e livre de danos para o paciente ${ }^{(15)}$.

\section{CONSIDERAÇÕES FINAIS}

Concluindo a presente revisão integrativa, na busca da literatura mais atual, em relação aos principais eventos adversos relacionados à terapia farmacológica pediátrica, entende-se que a tomada de decisão do enfermeiro sobre o aprazamento seguro e o preparo e a administração de fármacos, deve ser realizada baseada em avaliação criteriosa e individualiza de cada cliente. Para essa avaliação é necessário que o profissional esteja embasado cientificamente, para implementar intervenções eficazes que sejam capazes de reduzir e, até mesmo, evitar os eventos adversos oriundos da uni ou polifarmácia.

É preciso que o enfermeiro comece a pensar na IM como um EAM, pois quando o aprazamento dos fármacos prescritos é realizado de forma negligente e imprudente pode desencadear uma RAM, acarretando um período maior de internação do cliente e onerando todo o processo de hospitalização.
Diante do exposto, acredita-se que, para reduzir a ocorrência dos EAM nas unidades pediátricas, é preciso a adoção de algumas estratégias, como, por exemplo, a abordagem de tal assunto junto aos integrantes da equipe de enfermagem e médica em parceria com o setor de educação permanente. É preciso também que seja adotado nos setores pediátricos um manual contendo: nome genérico do medicamento, classificação terapêutica, dose máxima/dia, vias de administração, $\mathrm{pH}$, osmolaridade e incompatibilidade (droga-droga, droga-alimento e droga-material).

Nesse sentido, sugere-se que o enfermeiro atue também frente à equipe de enfermagem de modo a conscientizá-la da importância da comunicação escrita, a fim de procederem adequadamente às anotações, pois desta forma será possível a notificação das reações adversas medicamentosas (oriundas da droga ou da interação entre fármacos) ao serviço de farmacovigilância hospitalar e, posteriormente, à Agência Nacional de Vigilância Sanitária (ANVISA).

Avaliando a proposta inicial desta revisão, observam-se lacunas na investigação relacionadas aos eventos adversos que podem ocorrer durante a terapia medicamentosa pediátrica. Os fatores que intermediam essa lacuna escapam ao propósito desta revisão. No entanto, parece claro que, como é possível localizar publicações, as quais indicam a possibilidade de ocorrência de eventos adversos relacionados ao aprazamento da prescrição médica (interações medicamentosas), às reações adversas e erro no preparo e/ou administração dos fármacos, há a necessidade de o enfermeiro implementar, na prescrição de enfermagem, cuidados inerentes à terapia medicamentosa de cada cliente.

Frente às lacunas encontradas e aos resultados apontados no material incluído nessa revisão integrativa, entende-se ser necessário intensificar esforços para o desenvolvimento de pesquisas que visem, sobretudo, minimizar a ocorrência do erro e orientar a equipe de enfermagem quanto à maneira correta de intervir diante de eventos adversos imprevisíveis e não planejados. Estudos com delineamento que produzam evidências fortes e relativas ao tema investigado, principalmente na realidade da prática da enfermagem pediátrica.

\section{REFERENCIAS}

1. Leite SN, Vieira M, Veber AP. Estudos de utilização de medicamentos: uma síntese de artigos publicados no Brasil e América Latina. Ciência \& Saúde Coletiva. [serial online]. 2008 Apr [cited 2010 Jun 30];13(supl):793-802. Available from: http://redalyc.uaemex.mx/pdf/630/63009726.pdf

2. Mendes W, Travassos C, Martins M, Noronha JC. Revisão dos estudos de avaliação da ocorrência de eventos adversos em hospitais. Rev. bras. epidemiol. [serial online]. 2005 Dec [cited 2009 Oct 28];8(4):393-406. Available from: http://www. scielo.br/scielo.php?script $=$ sci arttext\&pid $=$ S1415-790X2 005000400008\&lng $=$ en\&nrm $=$ iso\&tlng $=p t$

3. Rosa MB, Perini E. Erros de medicação: quem foi? Rev. Assoc. Med. Bras.[serial online]. 2003 Sep [cited 2009 Nov 01];49(3):335-341. Available from: http://www.scielo.br/ scielo.php?pid = S0104-42302003000300041 \&script = sci arttext\&tlng $=\mathrm{es}$

4. Yamanaka TI, Pereira DG, Pedreira MLG, Peterlini MAS. Redesenho das atividades de enfermagem para redução de erros de medicação em pediatria. Rev Bras Enferm [serial online]. 2007 Apr [cited 2009 Oct 20];60(2):190-196. Available from: http://www.scielo.br/scielo.php?script =sci arttext\&pid $=$ S0034-71672007000200012\&lng $=$ en\&nrm $=$ iso\&tlng $=$ pt.

5. Impiccitore P, Choonara I, Clarkson A, Provasi D, Pandolfini $\mathrm{C}$, Bonati $\mathrm{M}$. Incidence of adverse drug reactions in paedriatric in/out-patients; a systematic review and meta-analysis of prospective studies. $\mathrm{Br} J$ Clin pharmacol 2001;52(2):77-83. 
6. Conroy S, McIntyre J, Choonara I, Stephenson T. Drug trials in children: problems and the way forward. Br J. Clin Pharmacol 2000; 49 (3): 93-97.

7. Galvão CM, Sawada NO, Rossi LA. Evidence-based practice: theoretical considerations on its implementation in perioperative nursing. Rev. Latino-Am. Enfermagem [serial online]. 2002 Oct [cited 2009 Feb 04];10(5):690-695. Available from: http://www.scielo.br/scielo.php?script=sci_art text\&pid $=$ S0104-11692002000500010.

8. Ingersoll GL. Evidence-based nursing: what it is and what it isn't. Nurs Outlook 2000;48(4):151-2.

9. Peterlini MAS, Chaud MN, Pedreira MLG. Órfãos de terapia medicamentosa: a administração de medicamentos por via intravenosa em crianças hospitalizadas. Rev. Latino-Am. Enfermagem [serial online]. 2003 Fev [cited 2009 Out 06];11(1):88-95. Available from: http://www.scielo.br/scielo.php?pid = S0104-11692003000100013\&script $=$ sci arttext.

10. Peterlini $M$, Chaud $M$, Pedreira M. Incompatibility among drugs and the materials of intravenous therapy catheters and disposable accessories. Descriptive study Online Brazilian Journal of Nursing [serial online]. 2006 December 16; [cited 2009 February 4];5(3):[about 5p.]. Available from: http:// www.uff.br/objnursing/index.php/nursing/article/view/483.

11. Lima REF, Cassiani SHB. Interações medicamentosas potenciais em pacientes de Unidade de Terapia Intensiva de um Hospital Universitário do Ceará [Dissertação]. Ribeirão Preto (SP): Escola de Enfermagem de Ribeirão
Preto, Universidade de São Paulo; 2007. Mestrado em Enfermagem.

12. Pelliciotti JSS, Kimura M. Qualidade de vida relacionada à saúde de profissionais de enfermagem e erros de medicação em Unidades de Terapia Intensiva [Dissertação]. São Paulo (SP): Escola de Enfermagem, Universidade de São Paulo; 2009. Mestrado em Enfermagem.

13. Optiz SP, Cassiani SHB. Sistema de medicação: análise dos erros nos processos de preparo e administração de medicamentos em um hospital de ensino [Tese]. Ribeirão Preto (SP): Escola de Enfermagem de Ribeirão Preto, Universidade de São Paulo; 2006. Doutorado em Enfermagem.

14. Coimbra JA, Cassiani SHB. Responsabilidade da enfermagem na administração de medicamentos: algumas reflexões para uma prática segura com qualidade de assistência. Rev. Latino-Am. Enfermagem [serial online]. 2001 Abr [cited 2009 Nov 01];9(2):56-60. Available from: http://www.scielo.br/scielo.php?script $=$ sci arttext\&pid $=$ S0104-11692001000200008\&lng $=$ pt\&nrm $=$ iso\&tlng $=$ pt.

15. Lima REF, Cassiani SHB. Interações medicamentosas potenciais em pacientes de unidade de terapia intensiva de um hospital universitário. Rev. Latino-Am. Enfermagem [serial online]. 2009 Abr [citado 2009 Out 14];17(2):222-227. Available from: http://www.scielo.br/scielo.php?script $=$ sci arttext\&pid = S0104-11692009000200013\&lng = en\&nrm $=$ iso\&tlng $=$ pt. 